\title{
Direction-Specific Adaptation in Neuronal and Behavioral Responses of an Insect Mechanosensory System
}

\author{
Hiroto Ogawa ${ }^{1,2}$ and Kotaro $0 \mathrm{ka}^{3}$ \\ ${ }^{1}$ Department of Biological Sciences, Faculty of Science, Hokkaido University, Sapporo 060-0810, Japan, ${ }^{2}$ PREST, Japan Science and Technology Agency, \\ Kawaguchi 332-0012, Japan, and ${ }^{3}$ Department of Bioscience and Informatics, Faculty of Science and Technology, Keio University, Yokohama 223-8522, \\ Japan
}

Stimulus-specific adaptation (SSA) is considered to be the neural underpinning of habituation to frequent stimuli and novelty detection. However, neither the cellular mechanism underlying SSA nor the link between SSA-like neuronal plasticity and behavioral modulation is well understood. The wind-detection system in crickets is one of the best models for investigating the neural basis of SSA. We found that crickets exhibit stimulus-direction-specific adaptation in wind-elicited avoidance behavior. Repetitive air currents inducing this behavioral adaptation reduced firings to the stimulus and the amplitude of excitatory synaptic potentials in wind-sensitive giant interneurons (GIs) related to the avoidance behavior. Injection of $\mathrm{a} \mathrm{Ca}^{2+}$ chelator into GIs diminished both the attenuation of firings and the synaptic depression induced by the repetitive stimulation, suggesting that adaptation of GIs induced by this stimulation results in $\mathrm{Ca}^{2+}$-mediated modulation of postsynaptic responses, including postsynaptic short-term depression. Some types of GIs showed specific adaptation to the direction of repetitive stimuli, resulting in an alteration of their directional tuning curves. The types of GIs for which directional tuning was altered displayed heterogeneous direction selectivity in their $\mathrm{Ca}^{2+}$ dynamics that was restricted to a specific area of dendrites. In contrast, other types of GIs with constant directionality exhibited direction-independent global $\mathrm{Ca}^{2+}$ elevation throughout the dendritic arbor. These results suggest that depression induced by local $\mathrm{Ca}^{2+}$ accumulation at repetitively activated synapses of key neurons underlies direction-specific behavioral adaptation. This input-selective depression mediated by heterogeneous $\mathrm{Ca}^{2+}$ dynamics could $^{2}$ confer the ability to detect novelty at the earliest stages of sensory processing in crickets.

Key words: calcium imaging; dendritic integration; directional selectivity; insect; stimulus-specific adaptation; synaptic depression

\section{Significance Statement}

Stimulus-specific adaptation (SSA) is considered to be the neural underpinning of habituation and novelty detection. We found that crickets exhibit stimulus-direction-specific adaptation in wind-elicited avoidance behavior. Repetitive air currents inducing this behavioral adaptation altered the directional selectivity of wind-sensitive giant interneurons (GIs) via direction-specific adaptation mediated by dendritic $\mathrm{Ca}^{2+}$ elevation. The GIs for which directional tuning was altered displayed heterogeneous direction selectivity in their $\mathrm{Ca}^{2+}$ dynamics and the transient increase in $\mathrm{Ca}^{2+}$ evoked by the repeated puffs was restricted to a specific area of dendrites. These results suggest that depression induced by local $\mathrm{Ca}^{2+}$ accumulation at repetitively activated synapses of key neurons underlies direction-specific behavioral adaptation. Our findings elucidate the subcellular mechanism underlying SSA-like neuronal plasticity related to behavioral adaptation.

\section{Introduction}

Habituation is the simplest form of learning and causes animals to become less responsive to repeated occurrences of a stimulus.

\footnotetext{
Received April 9, 2015; revised July 9, 2015; accepted July 15, 2015.

Author contributions: H.O. and K.O. designed research; H.O. performed research; H.O. analyzed data; H.O. and K.O. wrote the paper.

This work was supported by the Japan Science and Technology Agency, Precursory Research for Embryonic Science and Technology (PRESTO) (H.O.), the Japan Society for the Promotion of Science, and the Ministry of Education, Culture, Sports, Science and Technology of Japan (KAKENHI Grants 24120502, 26119501, and 26440176 to H.0.). We thank Ruriko Mitani for additional imaging experiments, Matasaburo Fukutomi for assistance with statistical analysis, and Matthew H. Dick for helpful comments on the manuscript.
}

Repeated stimuli with similar physical properties may cause a reduction in an animal's reflex, whereas a novel stimulus with properties different from the repeated stimulus can elicit a strengthened reflex in the habituated animal (Rankin et al., 2009). In nature, detecting and attending to novel stimuli is a crucial task of the nervous system. Stimulus-specific adaptation

Correspondence should be addressed to Hiroto Ogawa. Department of Biological Science, Faculty of Science, Hokkaido University, Kita 10-jyo, Nishi 8, Kita-ku, Sapporo 060-0810, Japan. E-mail: hogawa@sci.hokudai.ac.jp. DOI:10.1523/JNEUROSCI.1378-15.2015

Copyright $\odot 2015$ the authors $\quad 0270-6474 / 15 / 3511644-12 \$ 15.00 / 0$ 
(SSA) is the neural process responsible for novelty detection, in which neurons that have adapted to frequently occurring stimuli respond strongly to a novel stimulus (Ringo, 1996). SSA occurs in the visual (Movshon and Lennie, 1979; Saul and Cynader, 1989; Dragoi et al., 2000), auditory (Condon and Weinberger, 1991; Malone et al., 2002; Ulanovsky et al., 2003), and somatosensory (Derdikman et al., 2006; Katz et al., 2006) cortices of mammals. For example, after repeated delivery of a single-frequency tone, auditory cortical neurons preferentially respond to novel stimuli of different frequencies rather than to the same prior stimulus (Ulanovsky et al., 2003). Similarly, short-term exposure to adapting visual stimuli with specific orientations shifts the orientation preference tuning curve of cat visual cortical neurons (Dragoi et al., 2000, 2001). Cortical SSA has been proposed as a higher-order feature of sensory processing that is transmitted to subcortical structures via top-down circuits (Nelken and Ulanovsky, 2007). In addition, SSA-like plasticity in neuronal responses has been reported in a variety of animals (Reches and Gutfreund, 2008; Ponnath et al., 2013) including in invertebrates with simple CNSs (Givois and Pollack, 2000; Schul and Sheridan, 2006; Schul et al., 2012). Therefore, SSA is considered to be the universal neural basis for novelty detection during habituation. An integrative study on SSA in a simple nervous system for which a behavioral role has been described should provide a complete picture of SSA, including how SSA-like plasticity in individual neurons is involved in the habituation of animal behavior, as well as how the cellular mechanism by which synaptic plasticity discriminates between frequent and novel inputs functions. To address these questions, we adopted a comprehensive approach to cercalmediated walking behavior in the cricket, combining behavioral testing, electrophysiology, and optical imaging techniques.

Crickets demonstrate oriented escape behavior to air current stimuli that is mediated by the cercal sensory system, a mechanosensory system in orthopteran insects that detects surrounding air currents (Jacobs et al., 2008). The receptor organs involved are a pair of antenna-like appendages called cerci at the rear of the abdomen, each covered with 500-750 mechanosensory filiform hairs (Palka et al., 1977; Miller et al., 2011). Air currents in the animal's immediate environment move these hairs and activate receptor neurons. The response amplitude of each receptor depends on the velocity and direction of the air current (Shimozawa and Kanou, 1984; Landolfa and Miller, 1995). Mechanosensory afferents make synapses with a group of interneurons, including 8-10 pairs of identified giant interneurons (GIs) (Jacobs and Murphey, 1987). These GIs have unique dendrites within the terminal abdominal ganglion (TAG) and extend large-diameter axons to higher centers including thoracic and cephalic ganglia (Hirota et al., 1993). Like the sensory afferents, the GIs are also sensitive to the direction and dynamics of air currents (Miller et al., 1991; Theunissen and Miller, 1991). Depolarizing current injection into specific GIs modifies turning locomotion and evokes leg movement (Hirota et al., 1993; Gras and Kohstall, 1998) and selective ablation of single GIs alters the motor control of wind-elicited walking behavior (Oe and Ogawa, 2013). These observations imply a crucial role for GIs in this behavior. This makes it an ideal model for investigating the behavioral impact of SSA because cellular substrates in the underlying neural circuit have been identified and distinct roles for these cells and response properties, such as the directional selectivity, have been well documented. The aim of this study was to clarify the neuronal mechanism underlying direction-specific adaptation in the wind-elicited walking behavior in the cricket.

\section{Materials and Methods}

Animals. Laboratory-bred adult male crickets (Gryllus bimaculatus) (body length, 23-31 mm; body weight, $0.50-1.20 \mathrm{~g}$ ) were used in the experiments. The animals were reared under $12: 12 \mathrm{~h}$ light/dark conditions at a constant temperature of $27^{\circ} \mathrm{C}$. Behavioral experiments were conducted during the light phase at room temperature. The guidelines of the Institutional Animal Care and Use Committee of the National University Corporation Hokkaido University contain no specific instructions for the use of insects in experiments.

Treadmill system. Behavioral experiments were conducted with a spherical treadmill system as described in a previous study (Oe and Ogawa, 2013). An animal was tethered to the top of a Styrofoam ball ( $\varnothing=$ $60 \mathrm{~mm}, 2.3 \mathrm{~g}$ ) by means of a pair of insect pins bent into an $\mathrm{L}$ shape that were fixed with paraffin to the cricket's tergite. The head of each pin was inserted into a glass tube ( $\varnothing=1 \mathrm{~mm}$ ), enabling the cricket to lift its body and walk freely on the ball, which was floating on a stream of air (see Fig. $1 A)$. The treadmill was placed in the center of a circular experimental arena $24 \mathrm{~cm}$ in diameter. The cricket's locomotion was indicated by the rotation of the ball, monitored at a sampling rate of $100 \mathrm{~Hz}$ by means of two optical detectors mounted orthogonally on each side of the ball. TrackTaro software (Chinou Jouhou Shisutemu) was used to calculate the walking velocity based on the measured ball rotation. This experiment was performed in a dark, soundproof chamber and the cricket's behavior was also monitored with a CAM 130 Night Vision infrared charge-coupled device (CCD) video camera (Timely) at 30 frames/s.

Air current stimulation. Air puff stimuli consisted of a short puff of nitrogen gas from a plastic nozzle (diameter, $15 \mathrm{~mm}$ ) connected to a PV820 pneumatic picopump (World Precision Instruments). The velocity of the air puff was $\sim 1.0 \mathrm{~m} / \mathrm{s}$ measured at the cerci. The duration of each puff was $200 \mathrm{~ms}$ and the interpuff interval in repetitive stimulations was 1, 2, or $5 \mathrm{~s}$. Eight nozzles for stimulus delivery were arranged around the cerci in the same horizontal plane. The nozzle ends were positioned $75 \mathrm{~mm}$ from the animal, with a $45^{\circ}$ angle between neighboring nozzles. The stimulus angle was manually switched by means of airflow valves connected to the nozzles.

Behavioral test. To test for direction-specific adaptation in the windelicited walking behavior, we designed the following protocols of repetitive stimulation. In the $-90^{\circ}$ to $180^{\circ}$ protocol, the stimulus sequence consisted of four successive puffs delivered from a fixed angle $\left(-90^{\circ}\right)$, followed by a puff from an angle perpendicular to the previous stimulus $\left(180^{\circ}\right)$ and a final puff from the initial angle $\left(-90^{\circ}\right)$. In the $180^{\circ}$ to $-90^{\circ}$ protocol, the angles of the frequent and novel stimuli were switched. All stimuli were provided at $2 \mathrm{~s}$ intervals. Using the treadmill system, we measured the maximum walking speed in the $2 \mathrm{~s}$ between the successive puffs. For each animal, four to eight trials were performed for each of the two stimulation protocols. Trials were excluded if the walking speed in the response to the first puff was lower than $0.1 \mathrm{~m} / \mathrm{s}$.

Isolated preparation. We prepared a preparation of the cercal sensory system isolated from the body for electrophysiological recordings and optical imaging under a fluorescence microscope. After the head, wings, and legs of the cricket were removed, an incision was made along the dorsal midline of the abdomen. The gut, internal reproductive organs, and surrounding fat were removed to expose the TAG. Peripheral nerves of the TAG were severed except for the cercal nerves. To remove the sheath from the TAG and allow penetration of a glass microelectrode, a piece of filter paper soaked in 10\% type XIV protease (Sigma-Aldrich) was placed on the dorsal or ventral side. After washing with isotonic saline, the preparation, consisting of the sixth and terminal abdominal ganglia, abdominal connective nerves, cercal nerves, and cerci, was removed from the body and whole mounted in a glass recording chamber.

Electrophysiology. GI membrane potentials were recorded intracellularly with a glass microelectrode $(30-50 \mathrm{M} \Omega$ ) filled with $150 \mathrm{~mm}$ potassium acetate and $2 \mathrm{~mm}$ Oregon green 488 BAPTA-1 (OGB-1) potassium salt (Life Technologies). GI action potentials were also monitored with a pair of hook electrodes positioned under the abdominal connective nerve cords. To estimate the synaptic efficiency, paired pulses (interpulse interval, $20 \mathrm{~ms}$; pulse duration, $50 \mu \mathrm{s}$ ) of electrical stimulation were applied to the left or right cercal nerve with two pairs of hook electrodes 
at $1 \mathrm{~Hz}$. The stimulus intensity was adjusted to a level that evoked subthreshold EPSPs without generating action potentials. Electrophysiological signals were digitized at $20 \mathrm{kHz}$ with a Powerlab $4 \mathrm{~S}$ analog-to-digital converter (AD Instruments) and analyzed with Chart version 7 software (AD Instruments).

Fluorescent indicator loading and optical recordings. The fluorescent $\mathrm{Ca}^{2+}$ indicator (OGB-1) was loaded iontophoretically into GIs for $5 \mathrm{~min}$ through a glass microelectrode at a hyperpolarizing current of $3 \mathrm{nA}$. Fluorescence images were observed with an Axiovert 100 inverted microscope (Carl Zeiss) equipped with a Fluar $10 \times, 0.5$ numerical aperture dry objective lens (Carl Zeiss), with illumination from an XBO $75 \mathrm{w}$ Xenon arc lamp (Carl Zeiss) having a stabilized power supply and a $480 / 20$ band-pass filter for the excitation of OGB-1. GI Ca ${ }^{2+}$ signals were measured from fluorescence images captured through a FT510 dichroic mirror and 535/45 band-pass filter. A series of images was acquired at $30 \mathrm{~Hz}$ and $256 \times 256$ pixels with an ORCA-ER digital cooled-CCD camera (Hamamatsu Photonics) attached to the microscope and AQUACOSMOS/Ratio software (Hamamatsu Photonics), which was also used to measure the OGB-1 fluorescence intensity. Fluorescence intensities obtained from each image were corrected for background and the average value was determined for each polygonal-recording region of interest (ROI). To obtain time course displays of changes in fluorescence for several ROIs, the mean fluorescence intensities were plotted as a function of time. Changes in cytosolic $\mathrm{Ca}^{2+}$ concentration were calculated as $\Delta F / F[\Delta F / F=(F-$ $\left.\left.F_{0}\right) / F_{0}\right]$, where $F_{0}$ was the corrected prestimulus fluorescence intensity. Relative fluorescence $(\Delta F / F)$ images were processed and displayed in pseudocolor with AQUACOSMOS/Ratio software. A $3 \times 3$ pixel median filter was applied to each image presented.

Data analysis. To analyze the direction-selective properties of firing activity and dendritic $\mathrm{Ca}^{2+}$ signals, we calculated the directional tuning curves in which the number of spikes evoked or the amplitude of transient increase of $\mathrm{Ca}^{2+}$ in response to air puffs were plotted as a function of stimulus angle. The number of spikes and amplitudes of $\mathrm{Ca}^{2+}$ increase were each normalized to the maximum value among the responses to eight air currents delivered from different directions. The mean angle was used to characterize the directional bias in the spikes and $\mathrm{Ca}^{2+}$ responses and was defined in each as the angle of the mean vector of all response vectors evoked by the stimuli from different directions.

Statistical analysis. To assess the significance of behavioral adaptations, we used a one-way repeated-measures ANOVA when the responses to the first to the sixth puffs measured from the same individuals were compared based on the normalized each maximum walking velocity. If there was a significant difference, we compared the each response with the second to the sixth puff stimulus to the naive response to the first puff using a post hoc Dunnett's test. The maximum walking velocities were normalized to the average value of the responses to the first puff in each individual animal. To assess the significance of the adaptation in firing responses, a one-way factorial ANOVA was used when the responses recorded from different populations of the recorded neurons were compared in the number of spikes among the normalized responses to the second to the fifth puffs. If there was a significant difference, we compared the populations between all pairs of responses with the first to the fifth puff stimuli using a post hoc Tukey's test. The numbers of spikes in responses to the second to the fourth puffs were normalized to the average spike counts evoked by the first puff and the numbers in response to the fifth puff were normalized to the naive response to the stimulus from the same direction in each sample recorded. The magnitude of the naive response used to normalize the response to the fifth puff was adjusted based on the proportion of the response to the first puff to the naive response to the puff from the frequent stimulus direction.

To assess the significance of the directional tuning shift, we used a Watson-Williams test (Zar, 2010) when multiple populations were compared based on the mean angle calculated from the directional tuning curve among different conditions of adaptation (the naive and adapted conditions for the 1,2, and $5 \mathrm{~s}$ intervals of repeated stimuli). To assess the significance of the attenuation rate of firing responses, a two-way repeated-measures ANOVA was used when the responses recorded from the same neurons were compared based on the number of spikes nor- malized for the tuning curves between the stimulus angles and between the stimulus intervals.

To assess the significance of effects of the $\mathrm{Ca}^{2+}$ chelator, a MannWhitney $U$ test was used when the responses recorded from different populations of neurons were compared between the BAPTA-injected and dye-injected conditions. The amplitude and paired-pulse ratio of EPSPs evoked by the subthreshold electrical stimulation were normalized to the average for $40 \mathrm{~s}$ before the repeated air current stimulation in each measurement. These values were averaged for two different poststimulation periods $20-40 \mathrm{~s}$ and $60-80 \mathrm{~s}$ after the onset of the repetitive stimulation.

To evaluate the significant difference in the $\mathrm{Ca}^{2+}$ accumulation, a two-way repeated-measures ANOVA was used when the $\mathrm{Ca}^{2+}$ responses were compared based on the peak value of transient $\mathrm{Ca}^{2+}$ increase and on the basal $\mathrm{Ca}^{2+}$ level between the stimulus angles and between the stimulus intervals. The peak value of the $\mathrm{Ca}^{2+}$ transients was determined as maximum value of $\Delta F / F$ in each response to individual puffs and the basal $\mathrm{Ca}^{2+}$ level was determined as $\Delta F / F$ value just before the next puff with successive stimulation. The peak and basal $\mathrm{Ca}^{2+}$ levels were normalized to the corresponding values of the $\mathrm{Ca}^{2+}$ transient evoked by the first puff in each measurement. To evaluate the significant difference in $\mathrm{Ca}^{2+}$-buffering capacity, a Mann-Whitney $U$ test was used when the $\mathrm{Ca}^{2+}$ responses induced by a single action potential recorded from different populations of neurons were compared based on the time constant of the $\mathrm{Ca}^{2+}$ decay between two types of neurons (GIs 8-1 and 10-2). The numbers of animals sampled and measurements are stated in figure legends. Statistical tests were performed using R programming and ExcelTokei add-in software (SSRI).

\section{Results \\ Direction-specific adaptation in wind-elicited walking behavior}

To determine whether wind-elicited behavior in crickets exhibits SSA, air puff stimuli were delivered repeatedly to a cricket tethered on a floating ball in a spherical treadmill system (Fig. 1A), which allowed cricket locomotion, including walking velocity and angular velocity, to be monitored at high temporal resolution $(\sim 1 \mathrm{kHz})$ (Oe and Ogawa, 2013). During repetitive stimulation in the $-90^{\circ}$ to $180^{\circ}$ protocol (see Materials and Methods), the cricket began walking in response to each puff and turned in the clockwise direction to avoid the stimulus (Fig. 1B). The walking velocity gradually decreased throughout the delivery of the first four air puffs and then recovered slightly in response to the fifth air puff from an orthogonal direction (Fig. 1C). The maximum velocity of each burst of running evoked by air puffs was decreased with repeated stimulation (Fig. $1 D ; p=6.58 \mathrm{E}-05$, oneway repeated-measures ANOVA); for example, the walking elicited by the fourth puff was significantly slower than that in the naive response to the first puff (Fig. $1 D$, top; $p=0.0086$, Dunnett's test). However, no significant adaptation was observed in the responses to the fifth puff from a different angle $(p=0.9116)$. The response to the final puff from the initial direction was lower than the naive response ( $p=0.0033$ ), indicating that the animal had adapted to air puff stimuli from the direction most frequently experienced. The $180^{\circ}$ to $-90^{\circ}$ protocol experiments, in which stimulus angles for the frequent (first to fourth and sixth puffs) and novel (fifth puff) stimuli were switched (Fig. 1D, bottom), similarly showed significant adaptation to the frequent stimulus ( $p=0.0360,0.0128$ for fourth and sixth puffs, respectively), although transient recovery of the response to the novel stimulus was not significantly different from the naive response $(p=$ 0.2198 for fifth puff). These results indicate that repetitive stimuli from a fixed angle induce stimulus-direction-specific adaptation in wind-elicited walking behavior. 
A

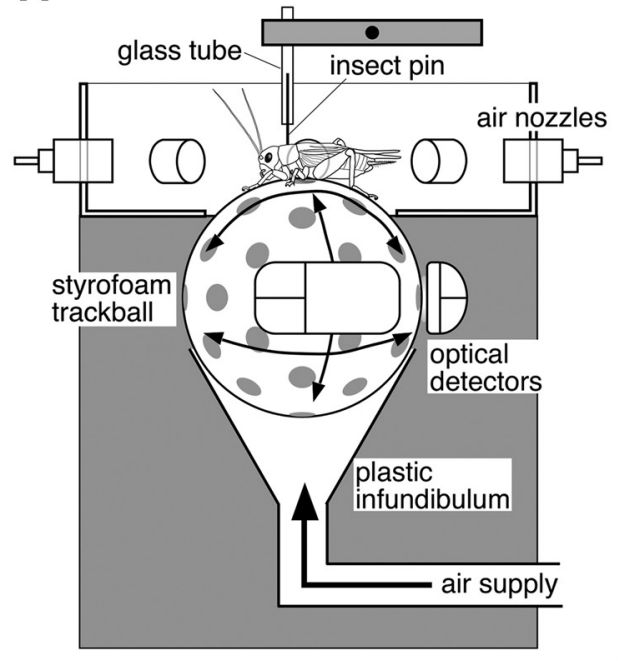

B

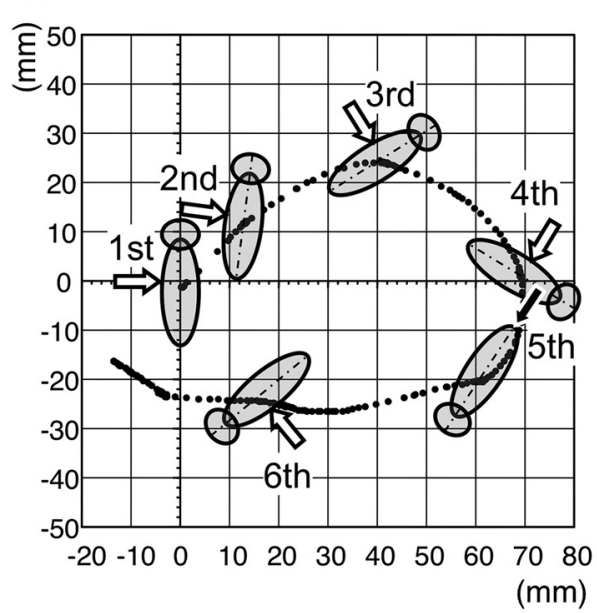

C
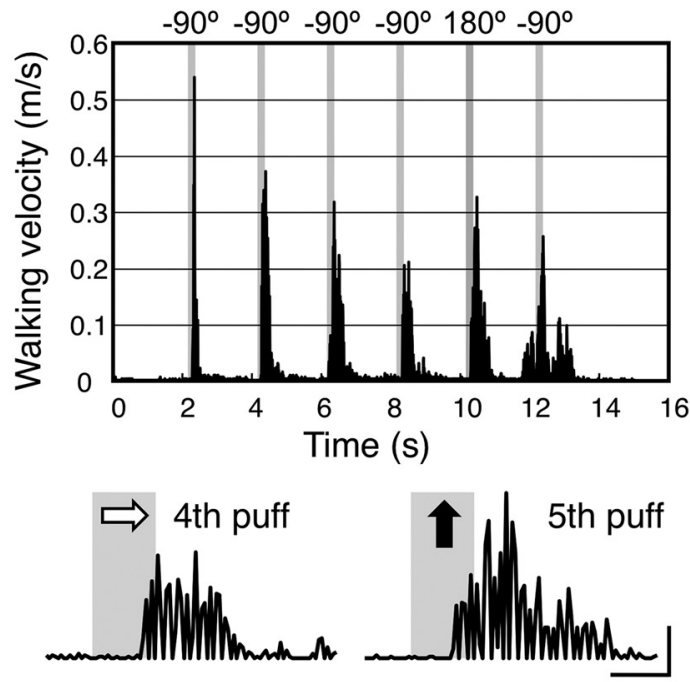

D

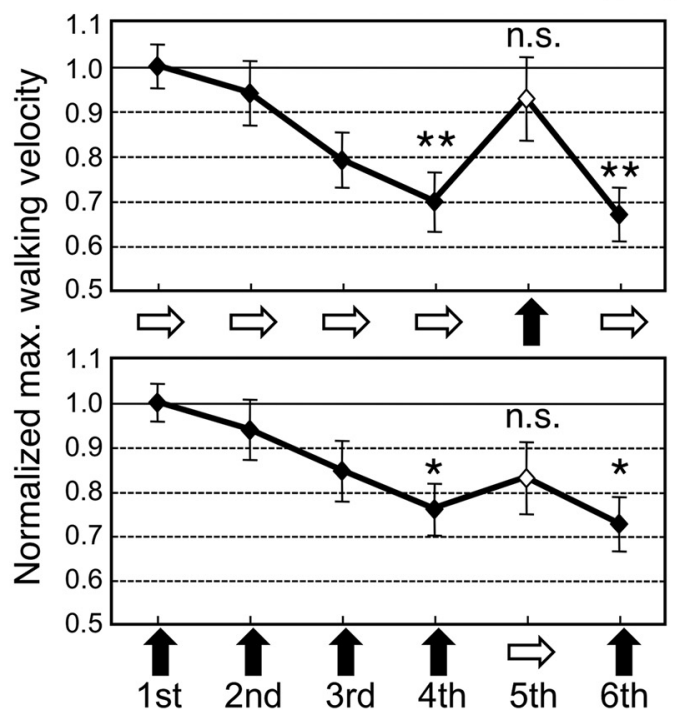

Figure 1. Stimulus-direction-specific adaptation of wind-elicited walking behavior in the cricket. $A$, Schematic representation of the spherical treadmill and air current stimulator. The cricket was tethered with insect pins inserted into a glass tube that allowed it to walk on a floating Styrofoam ball with two optical detectors monitoring the rotation. $\boldsymbol{B}$, Walking trajectory and body axis in response to repeated air puff stimulation. Puffs 1 to 4 and 6 were delivered from the left side $\left(-90^{\circ}\right)$, whereas number 5 was delivered from behind $\left(180^{\circ}\right)$. Each dot shows the central point of the body measured at $10 \mathrm{~ms}$ intervals. Arrows indicate the angle of each puff with respect to the animal. $\boldsymbol{C}$, Temporal profile of translational velocity during the walking behavior shown in $\boldsymbol{B}$. At the bottom are magnified traces of the velocity during walking elicited by the fourth (left) and fifth puffs. Vertical scale bar, $0.1 \mathrm{~m} / \mathrm{s}$; horizontal scale bar, $200 \mathrm{~ms}$. The duration of each puff is highlighted in gray. $\boldsymbol{D}$, Adaptation curves at maximum translational velocity during brief walking elicited by each puff of repetitive stimulation. At the top are data for the response to iterative puffs from $-90^{\circ}$ and a single pufffrom $180^{\circ}$. At bottom are data from an experiment in which the iterative and novel stimuli angles were switched. The responses to the iterative stimuli are indicated by filled lozenges and those to the novel stimuli (fifth puff) by open lozenges. The walking velocity was significantly altered depending on the stimulus order $\left(p=6.58 \mathrm{E}-05,46\right.$ trials for $-90^{\circ}$ to $180^{\circ}$ protocol; $p=$ $0.0052,47$ trials for $180^{\circ}$ to $-90^{\circ}$ protocol; $n=13$ crickets; one-way repeated-measures ANOVA). Asterisks mark walking responses in which the maximum velocity was significantly slower than the naive response to the first puff ( ${ }^{*} p<0.05,{ }^{* *} p<0.01$, Dunnett's post hoc test). Pooled data in $\boldsymbol{D}$ are represented as the mean \pm SEM.

\section{Direction-specific adaptation in wind-evoked responses in GIs}

We next investigated whether repeated puff stimulation inducing direction-specific behavioral adaptation modulates the directional sensitivity of individual GIs involved in wind-elicited behaviors. As in the behavioral experiments, four short (200 ms) puffs were applied from an angle contralateral to the GI cell body $\left(-90^{\circ}\right)$, followed by a fifth puff applied from the posterior direction $\left(180^{\circ}\right)$. GIs $8-1$ and $10-2$ generated a burst of action potentials in response to each puff (Fig. 2A). Both types of GIs showed adaptation to repeated stimulation: a stepwise reduction in the response to each puff was evident in both the number of spikes and the amplitude of EPSPs. In GI 8-1, however, the fifth puff applied from an angle of $180^{\circ}$ elicited firings as vigorous as the naive response, indicating that GI 8-1 had selectively adapted to the angle from which the repetitive stimulus was delivered. As the interpuff interval was de- creased, the firing in response to each of the second to the fourth puffs was attenuated in GI 8-1 (Fig. $2 B ; p=9.463 \mathrm{E}-15$, $4.104 \mathrm{E}-22$ and $1.329 \mathrm{E}-33$ for 5,2 , and $1 \mathrm{~s}$ intervals, respectively; one-way factorial ANOVA), implying that the degree of attenuation of the firing depended on the stimulus interval. After the attenuation resulting from repeated puffs delivered at $5 \mathrm{~s}$ intervals, the firing to the fifth puff from another direction was recovered ( $p=0.0031$ between third and fifth puffs, $p=0.0011$ between fourth and fifth puffs, Tukey's test). Firing recovery was also observed for the 2 and 1 s intervals, but there was no significant difference between firings to the fourth and fifth puffs ( $p=0.11175,0.27611$ for 2 and $1 \mathrm{~s}$ intervals, respectively). The adaptation induced by repetitive stimulation at higher frequency was probably too suppressive to allow the recovery of firings to the novel stimulus for GI 8-1. 


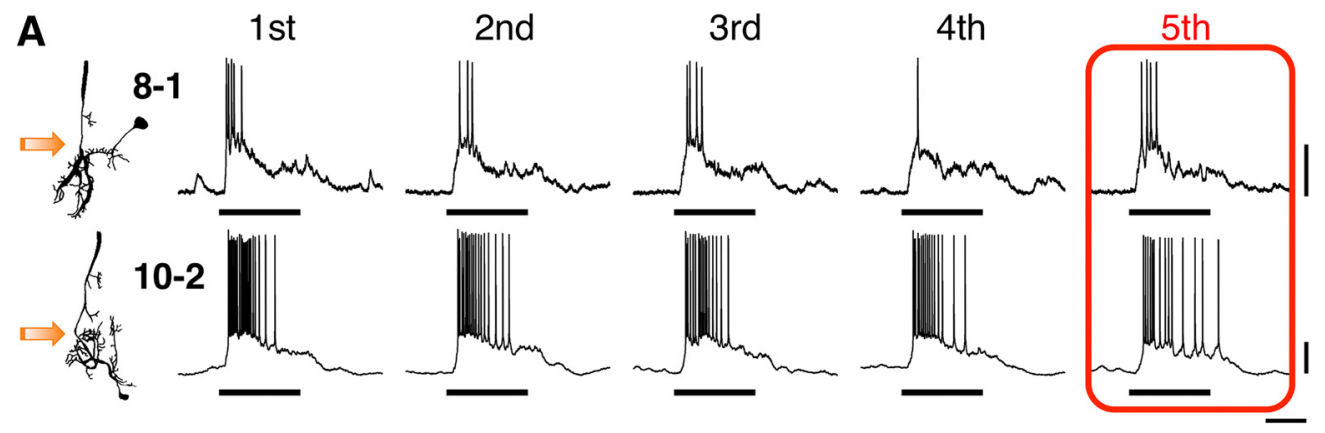

B

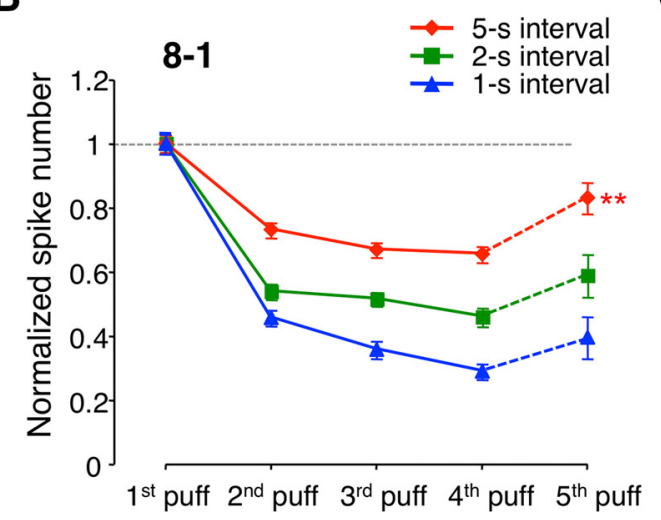

C

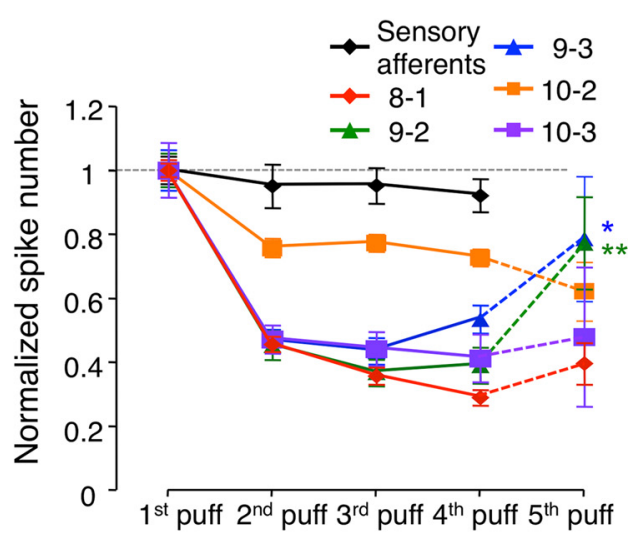

Figure2. Attenuation in GI responses to each puff during repetitive stimulation. $A$, Example of the burst response to each puff during repetitive stimulation. The first to fourth puffs were delivered from the side contralateral to the soma $\left(-90^{\circ}\right.$; orange arrow), whereas the fifth puff was delivered from the posterior side $\left(180^{\circ}\right)$. Black bars indicate the stimulus duration. Vertical scale bars, 20 $\mathrm{mV}$; horizontal scale bar, $100 \mathrm{~ms} . \boldsymbol{B}, \boldsymbol{C}$, Changes in spike number in the burst response during repetitive stimulation. The first to fourth puffs were delivered from the side contralateral to the soma $\left(-90^{\circ}\right)$, whereas the fifth puff was delivered from various angles other than $-90^{\circ}$. B. Dependency of the adaptation on the interpuff interval in GI 8-1. The firing responses to each puff were significantly reduced depending on the stimulus order during the repetitive stimulation at all interpuff intervals ( $p=9.463 \mathrm{E}-05,36$ trials in $5 \mathrm{Gls}$ for $5 \mathrm{~s}$ intervals; $p=4.104 \mathrm{E}-22,40$ trials in $6 \mathrm{Gls}$ for 2 s intervals; $p=1.329 \mathrm{E}-33,43$ trials in $6 \mathrm{Gls}$ for 1 s intervals; one-way factorial ANOVA). The numbers of spikes evoked by the first puff used for normalization in $\boldsymbol{B}$ were $6.98 \pm 0.97,5.18 \pm$ 0.49 , and $4.57 \pm 0.21$ for the 5, 2, and $1 \mathrm{~s}$ interval experiments, respectively. C, Adaptation curves for mechanoreceptor afferents and for various types of Gls. The repeated puffs were delivered at $1 \mathrm{~s}$ intervals. The firing responses of all types of Gls were significantly reduced during the repetitive stimulation, but were not altered in the mechanoreceptor neurons $(p=8.434 \mathrm{E}-10,17$ trials in 3 neurons for GI 9-2; $p=2.198 \mathrm{E}-05,8$ trials in 1 neuron for GI 9-3; $p=2.974 \mathrm{E}-10$, 42 trials in 7 neurons for Gl 10-2; $p=0.0005,8$ trials in 1 neuron for Gl 10-3; $p=0.3682,22$ trials in 6 neurons for mechanosensory afferents; one-way factorial ANOVA). The spike numbers in the response to the first puff used for normalization in ( were 9.79 \pm 3.19 for Gl 9-2,7.5 for Gl 9-3, 20.37 \pm 4.36 for GI 10-2, and 4.25 for GI 10-3, $22.16 \pm 4.10$ for sensory afferents. Asterisks mark responses to the fifth puff that were significantly larger than those to the second, third, or fourth puff $\left({ }^{*} p<0.05\right.$, ${ }^{* *} p<0.01$, Tukey's post hoc test). Pooled data in $\boldsymbol{B}$ and $($ are represented as the mean \pm SEM.

Repetitive stimulus-induced attenuation was observed in all GI types recorded, including 8-1, 9-2, 9-3, 10-2, and 10-3. The repeated stimulation decreased the puff-evoked spikes of the GIs $(p=2.198 \mathrm{E}-05,8.434 \mathrm{E}-10,2.978 \mathrm{E}-10$, and 0.0005 for GIs $9-2$, 9-3, 10-2, and 10-3, respectively; one-way factorial ANOVA), but had no effect on the response of mechanoreceptor afferents (Fig. $2 C ; p=0.3682$ ). These results demonstrated that the attenuation of the GI response was not due to the adaptation of the primary mechanoreceptor neurons. Furthermore, the attenuation rate was dependent on GI type: the response of GI 8-1 was rapidly attenuated, whereas GI 10-2 adapted slowly to stimuli applied at similar intervals, suggesting that the attenuation involves postsynaptic properties that vary between GI types. There was also a difference in the recovery rate of the response to the novel stimulus direction (fifth puff) among the types of GIs. For example, similarly to GI 8-1, GIs 9-2 and 9-3 showed recovery in response to the fifth puff from the adapted response to the third or fourth puff ( $p=0.0033,0.0354$ for GIs 9-2 and 9-3, respectively; Tukey's test), whereas the response in GI 10-2 remained attenuated or decreased further $(p=0.1815)$. These results indicated that whether the adaptation to repeated stimuli is direction specific or direction nonspecific depended on the type of GIs.

\section{Repeated stimulation shifts directional tuning in GI 8-1 but not in 10-2}

To determine whether attenuation of the response induced by repetitive air puffs is direction specific or direction nonspecific, we examined the directional sensitivity to subsequent puff stimuli. In this experiment, four short puffs were delivered from a $-90^{\circ}$ angle contralateral to the GI cell body (Fig. $3 A$ ) at various intervals; the fifth puff was delivered from various directions. In GI 8-1, wind-evoked responses were attenuated across stimulus angles, but the preferred angle shifted from $-135^{\circ}$ to $180^{\circ}$ in response to puffs applied at 1 or $2 \mathrm{~s}$ intervals (Fig. 3A, left). Circular statistical analysis of the mean angle calculated from the tuning curves (see Materials and Methods) showed a significant difference between naive and adapted responses in GIs 8-1 (Fig. 3B, left; $p=0.0490$, Watson-Williams test) and 9-2 (Fig. $3 B$, right; $p=0.0181$ ); that is, repeated stimulation from a fixed angle altered the directional tuning of GIs 8-1 and 9-2.

When the stimulus was delivered from $-90^{\circ}$, which was coincident with the direction of the repetitive puffs, the response was attenuated to a greater degree than when the delivery was from other directions such as $-135^{\circ}$ or $180^{\circ}$. The greater the difference in the stimulus angle between the novel and repetitive stimuli, the less attenuation was observed in the GI 8-1 resp- 

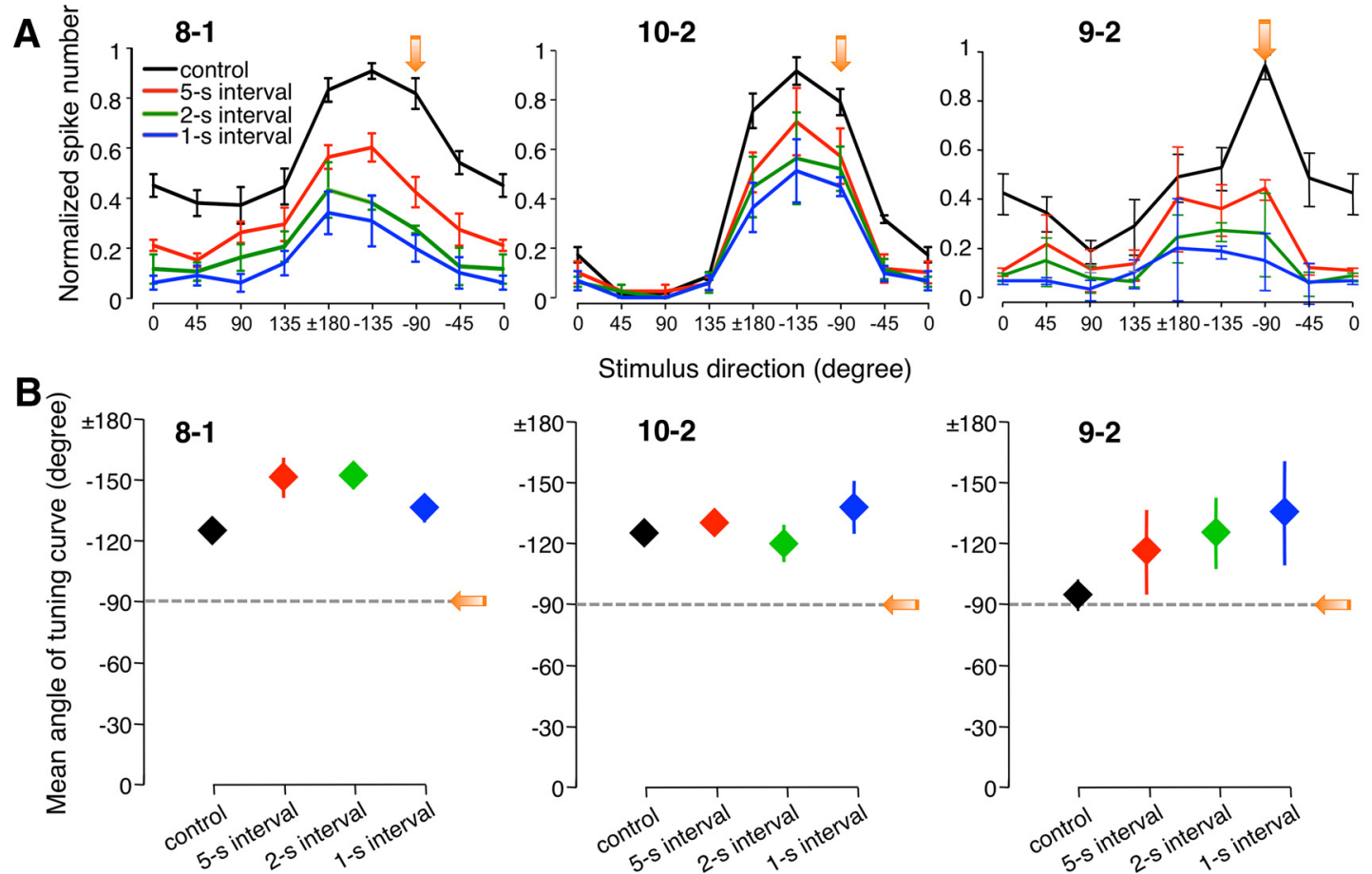

Stimulus direction (degree)
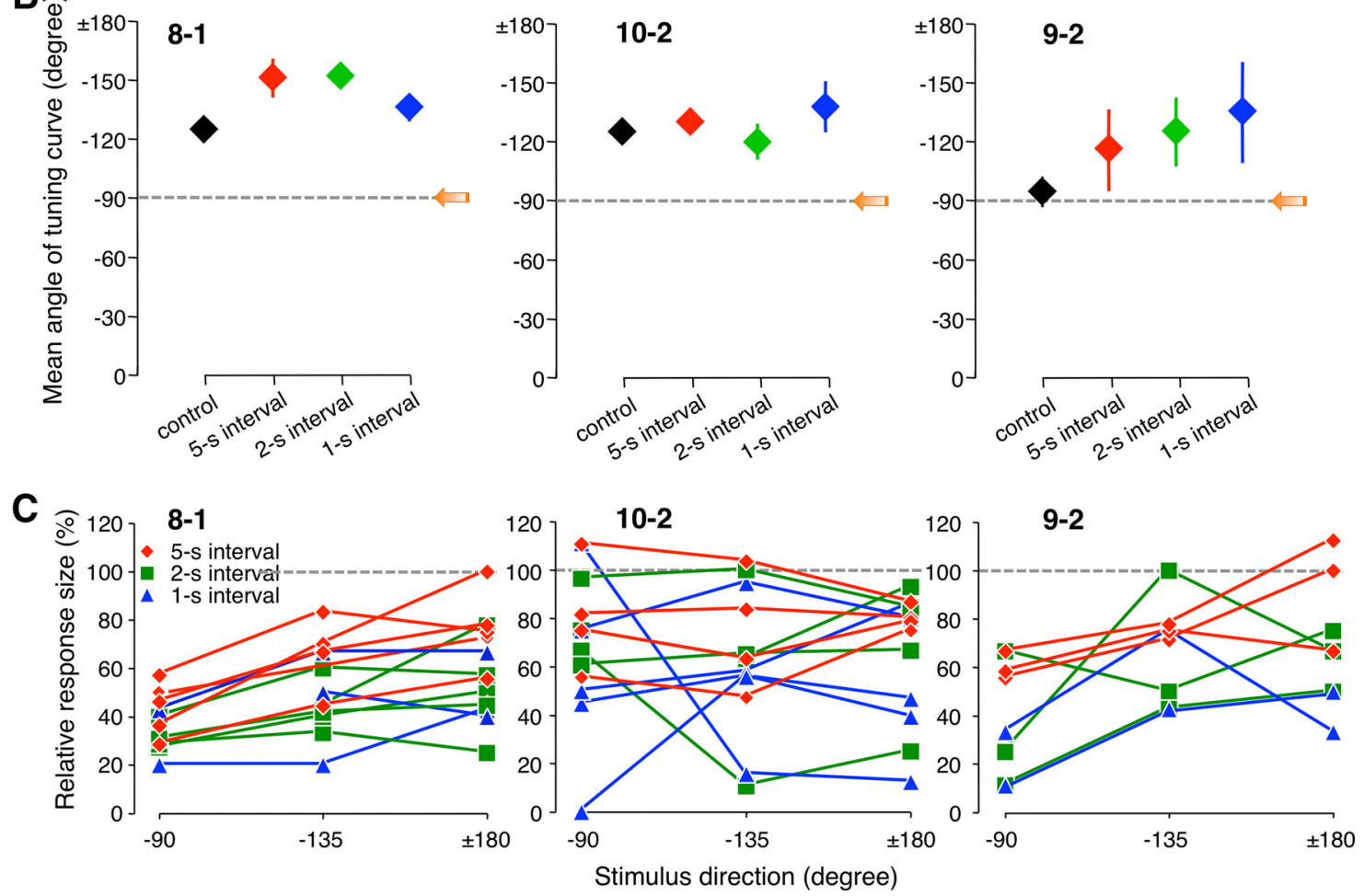

Figure 3. Directional tuning shift in Gls induced by repetitive stimulation. $A$, Directional tuning curves for Gls 8-1, 10-2, and 9-2 under control and adapted conditions. Red, green, and blue lines represent tuning curves after four puffs were repeatedly applied from the side contralateral to the soma (orange arrow) at 5,2 , and $1 \mathrm{~s}$ intervals, respectively. $\boldsymbol{B}$, Mean angles representing the directional tuning properties. Dashed lines indicate the stimulus angle of the repeated puffs $\left(-90^{\circ}\right)$. There were significant differences in mean angles among the control and adaptation conditions in Gls 8-1 and 9-2, but not in Gl 10-2 ( $p=0.0490,0.0181$, and 0.580 for Gls 8-1, 9-2, and 10-2, respectively, Watson-Williams test). C, Adapted response size in spike counts relative to the control (100\% is indicated by dashed line). Each line connecting the plots represents the data obtained from a single neuron. The attenuation rates in the spike counts depended on the stimulus angle in $\mathrm{Gls}$ 8-1 and 9-2, but not in Gl 10-2 ( $p=3.35 \mathrm{E}-06,0.00986$, and 0.671 for Gls 8-1, 9-2, and 10-2, respectively, two-way repeated-measures ANOVA). The data in this figure were obtained from 23 trials in 7 neurons for $\mathrm{Gl} 8-1,22$ trials in 6 neurons for $\mathrm{Gl} 10-2$, and 16 trials in 6 neurons for $\mathrm{Gl}$ 9-2. The spike counts in the maximum response used for normalization in $\boldsymbol{A}$ were $9.36 \pm 1.18,35.88 \pm 7.18$, and $11.00 \pm 2.09$ for Gls 8-1, 10-2, and GI 9-2, respectively. Pooled data in $\boldsymbol{A}$ and $\boldsymbol{B}$ are represented as the mean \pm SEM.

onse (Fig. 3C, left; $p=3.35 \mathrm{E}-06$, two-way repeated-measures ANOVA). In addition, there was a significant difference in the attenuation rate among the intervals of the repeated stimuli ( $p=$ $0.0188)$. These results demonstrate that attenuation was induced in a direction-specific manner and depended on the stimulus intervals in GI 8-1. This difference in the attenuation rate depending on the stimulus angle and on the stimulus intervals was also observed in GI 9-2 (Fig. 3C, right; $p=0.00986$ for stimulus angle and 0.0165 for stimulus interval, respectively, two-way repeated-measures ANOVA). In contrast, in GI 10-2, the preferred angle was not modulated by repeated stimulation; the directional profile of the attenuated response was like that of the naive response (Fig. $3 A, B$, middle; $p=0.5809$, Watson-Williams test) because the magnitude of the attenuation was constant regardless of stimulus angle or interval (Fig. $3 C$, middle; $p=0.671$ for stimulus angle and $p=0.124$ for stimulus interval).
Cytosolic $\mathrm{Ca}^{2+}$ mediates synaptic depression and attenuation of direction-specific responses

Differences between GIs 8-1 and 10-2 in directional tuning responses to repeated stimulation suggest that postsynaptic modulation of transmission efficiency at mechanoreceptor afferent-to-GI synapses is related to direction-specific or direction-nonspecific attenuation. To confirm that synaptic plasticity occurred during attenuation, EPSPs evoked by subthreshold stimulation of the cercal nerve were measured before and after the delivery of repeated air puffs. In response to 10 successive puffs applied at $1 \mathrm{~s}$ intervals, the EPSP amplitude was reduced to $70-80 \%$ for $>1 \mathrm{~min}$ (Fig. $4 A$ ). That is, repeated stimulation attenuated the GI response via short-term synaptic depression at cercal-to-GI synapses. In contrast, the paired-pulse ratio, which describes presynaptic changes, was unaltered by repeated stimulation. This suggests that the syn- 
A

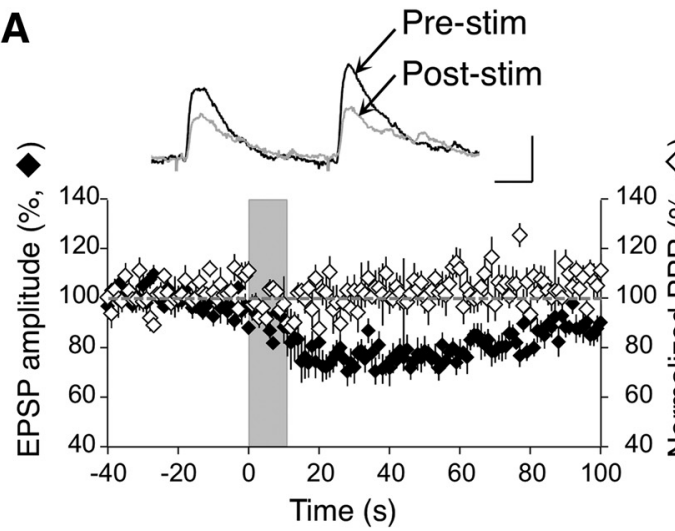

C

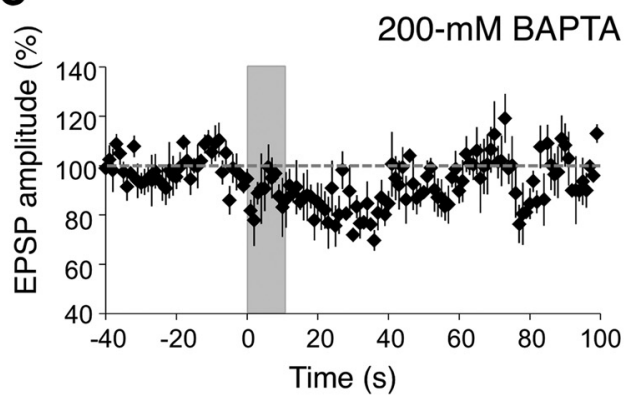

B

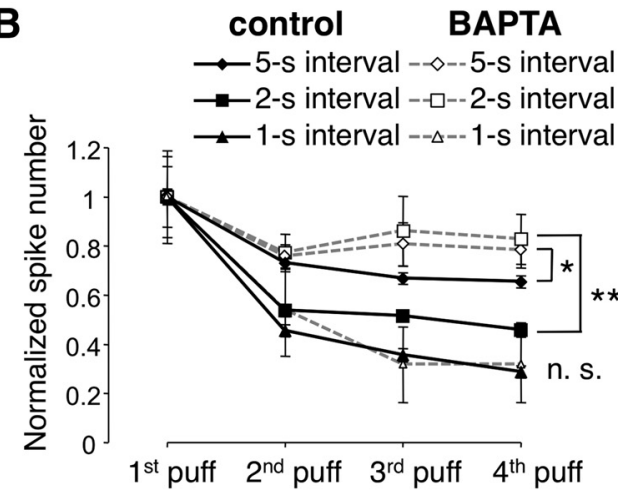

D

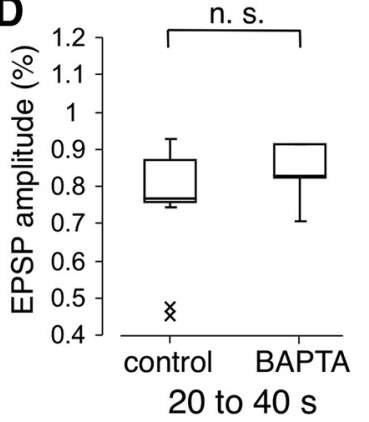

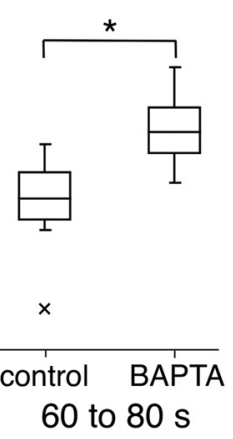

Figure 4. Synaptic plasticity induced by repetitive puff stimulation. $A$, Changes in EPSP amplitude and paired-pulse ratio measured in GIs before and after the delivery of 10 air puffs from the side contralateral to the soma at $1 \mathrm{~Hz}$ (gray bar). Top traces show representative EPSPs evoked by paired test pulses. Vertical scale bar, $5 \mathrm{mV}$; horizontal scale bar, $5 \mathrm{~ms}$. Data in $\boldsymbol{A}$ were 0 btained from 12 Gls. The amplitude of EPSPs used for normalization in $A$ was $8.786 \pm 0.946 \mathrm{mV}$. B, Effect of BAPTA injection on the adaptation induced by repeated puff stimulation in GI 8-1. BAPTA suppressed the attenuation of the response to the fourth puff during stimulation at 5 and 2 intervals $(p=0.0433,0.001$, and 0.6112 for the 5,2 , and 1 s intervals, respectively, Mann-Whitney $U$ test). The pooled data for the BAPTA-injected condition in $\boldsymbol{B}$ were obtained from 10 trials in $2 \mathrm{Gls}$ for each experiment with 5 and 2 sintervals and 6 trials in $2 \mathrm{Gls}$ for the 1 s interval experiments. The control data were obtained from the same Gls as used for the experiments shown in Figure 2B. C, Changes in EPSP amplitude in BAPTA-injected Gls before and after the delivery of repeated stimulation (10 puffs) at $1 \mathrm{~Hz}$ during the period indicated by the gray bar. Data in $\boldsymbol{C}$ were obtained from $5 \mathrm{Gls}$. The amplitude of EPSPs used for normalization in $\boldsymbol{A}$ was $7.494 \pm 1.132 \mathrm{mV}$. The pooled data in $\boldsymbol{A}-\boldsymbol{C}$ are represented as the mean \pm SEM. $D$, Effect of BAPTA injection on synaptic depression induced by repeated puff stimulation. The asterisk indicates a significant difference in EPSP amplitude between dye-injected (control) and BAPTA-injected Gls ( ${ }^{*} p<0.05$, Mann-Whitney $U$ test). The box plot whiskers indicate the $1.5 \times$ interquartile range of the lower and upper quartiles; box limits indicate the lower, median, and upper quartile from bottom to top; " $\times$ " indicates outlier data. The pooled data in $\boldsymbol{D}$ were obtained from the same $G l s$ used for the data in $\boldsymbol{A}$ and $\boldsymbol{C}$.

aptic depression induced by repetitive puffs results, not from the decrement of neurotransmitter release, but rather from postsynaptic modulation of receptor sensitivity or membrane properties.

The induction of synaptic plasticity at postsynaptic sites is largely triggered by the elevation of cytosolic $\mathrm{Ca}^{2+}$, which is a critical second messenger that triggers signaling cascades and protein phosphorylation (Zucker, 1999). Dendritic $\mathrm{Ca}^{2+}$ elevation modulates the responsiveness of cricket auditory neurons (Sobel and Tank, 1994; Baden and Hedwig, 2007) and mediates synaptic depression at the cercal-to-GI synapse (Ogawa et al., 2001). A recent study indicated that a $\mathrm{Ca}^{2+}$ mediated dendritic mechanism contributed to SSA in katydid auditory neurons (Triblehorn and Schul, 2013). To examine the role of $\mathrm{Ca}^{2+}$ in direction-specific attenuation, we injected the $\mathrm{Ca}^{2+}$ chelator BAPTA into GIs responding to repetitive puff stimulation. The concentration of BAPTA injected was $200 \mathrm{~mm}$, which is four times higher than that required to suppress dendritic $\mathrm{Ca}^{2+}$ elevation in GIs (Ogawa et al., 2001). The attenuation in responses to puffs delivered at 5 or $2 \mathrm{~s}$ intervals was diminished by the BAPTA, whereas the attenuation of spike counts in the instantaneous response to $1 \mathrm{~s}$ stimulation was unaffected (Fig. $4 B ; p=0.0370,0.0001$, and 0.7345 for 5,2 , and $1 \mathrm{~s}$ intervals, respectively, Mann-Whitney $U$ test). In BAPTA-injected GIs, synaptic depression induced by the $1 \mathrm{~s}$ puffs recovered more rapidly to pre stimulus levels
(Fig. 4C). The relative amplitude of EPSPs evoked in BAPTAinjected GIs was larger than in control (dye-injected) GIs during the 60-80 s poststimulation period (Fig. $4 D ; p=0.0265$, Mann-Whitney $U$ test). Postsynaptic $\mathrm{Ca}^{2+}$ elevation in dendrites thus plays an important role in the attenuation of the wind-evoked response and in sustaining synaptic depression induced by repeated air puff stimulation in the GIs. However, it remains unclear how the increase in postsynaptic $\mathrm{Ca}^{2+}$ selectively reduces synaptic inputs from sensory afferents sensitive to the direction of the repeated stimulus. We hypothesize that direction-specific or direction-nonspecific attenuation induced by repeated stimulation from a fixed angle is due to differences in the spatiotemporal dynamics of dendritic $\mathrm{Ca}^{2+}$ elevation in GIs.

\section{Directionality in dendritic $\mathrm{Ca}^{2+}$ responses}

Previous $\mathrm{Ca}^{2+}$-imaging studies have demonstrated that dendritic $\mathrm{Ca}^{2+}$ responses in GIs display unique directional tuning properties (Ogawa et al., 2004, 2008), suggesting that dendritic $\mathrm{Ca}^{2+}$ accumulation might vary in its spatial pattern depending on the stimulus direction. To explore the directional tuning properties of local dendritic $\mathrm{Ca}^{2+}$ responses in GIs 8-1 and 10-2, we visualized cytosolic $\mathrm{Ca}^{2+}$ changes in vivo during air current stimulation from various directions and examined the evoked $\mathrm{Ca}^{2+}$ signals in various regions across the entire dendritic arbor. Figure $5 A$ shows typical $\mathrm{Ca}^{2+}$ responses to the air current stimuli in three different dendritic regions 

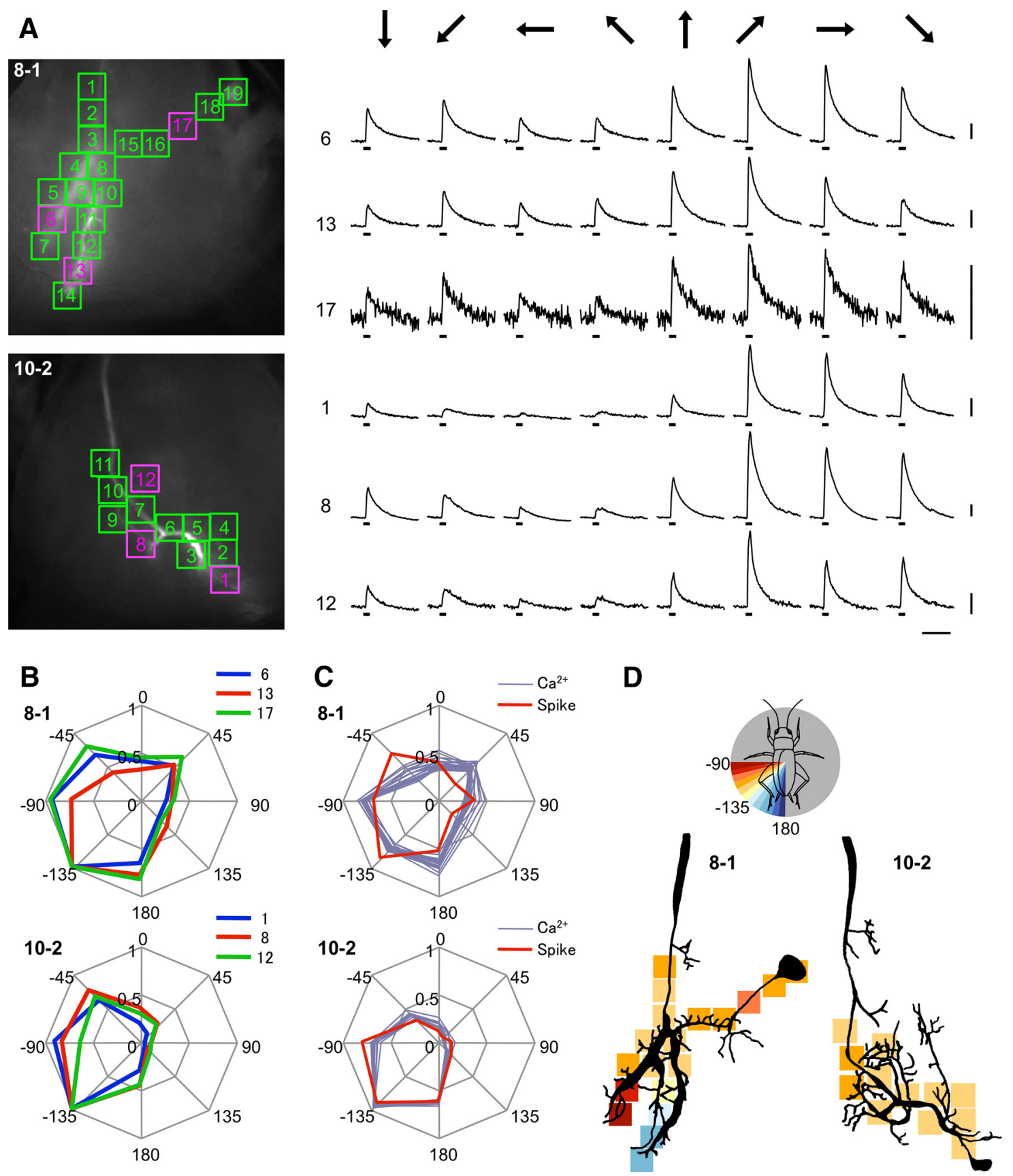

Figure 5. Directional tuning of local dendritic $\mathrm{Ca}^{2+}$ responses to air current stimuli in $\mathrm{Gls} . A, \mathrm{Ca}^{2+}$ responses in three different dendritic regions to air puff stimuli from eight different directions. Single short puffs with the duration indicated by black bars below each trace were delivered from the eight different directions indicated by arrows above the traces. Top three traces were recorded from GI 8-1, and the bottom three traces from GI 10-2. Left insets show fluorescence images of the Gls on which 19 or 12 ROls are superimposed. The number to the left of each trace indicates the ROI. Vertical scale bars, $5 \%$ in $\Delta F / F$; horizontal scale bar, $2 \mathrm{~s}$. B, Polar plots showing the directional tuning curves of $\mathrm{Ca}^{2+}$ responses in the three different ROls illustrated for each $\mathrm{Gl}$ in $A$. C, Directional tuning curves based on pooled data for dendritic $\mathrm{Ca}^{2+}$ responses in all ROls (gray lines, 6 trials in 3 neurons for Gl 8-1, 9 trials in 5 neurons for $\mathrm{Gl}$ 10-2) and for spike counts (red lines, 11 trials in 6 neurons for GI 8-1, 9 trials in 6 neurons for GI 10-2). D. Color maps indicating the spatial distribution of mean angles calculated from the directional tuning curves of $\mathrm{Ca}^{2+}$ signals. Color tiles superimposed on the drawings of the Gls represent the location of the mean angle. The inset diagram shows the color codes for mean angle with respect to the cricket body.

of GIs 8-1 (top three traces) and 10-2 (bottom three traces). We placed 19 (for GI 8-1) or 12 (for GI 10-2) ROIs on whole neurites, including dendritic branches (Fig. $5 A$, left insets). In all ROIs, the air current stimulus induced a transient increase in OGB-1 fluorescence. The amplitude of the $\mathrm{Ca}^{2+}$ increase depended on the stimulus directions, meaning that the $\mathrm{Ca}^{2+}$ responses in the GIs had distinct directional selectivity to the air current stimuli. Based on this result, we analyzed the directional tuning characteristics at individual subcellular locations. In the polar plots in Figure $5 B$, which indicate the directional tuning curves measured from three ROIs shown in Figure $5 \mathrm{~A}$, the amplitude of the transient increase in $\mathrm{Ca}^{2+}$ are plotted according to stimulus direction. These plots showed that the preferred directions in dendritic $\mathrm{Ca}^{2+}$ response were basically at the same angle $\left(-135^{\circ}\right)$, but the shapes of the tuning curves were different. Population data for the tuning curves based on the $\mathrm{Ca}^{2+}$ and spike responses show that the directional tuning curves were more various in GI 8-1, but similar to each other and close to the spike tuning curve in GI 10-2 (Fig. 5C). 
A

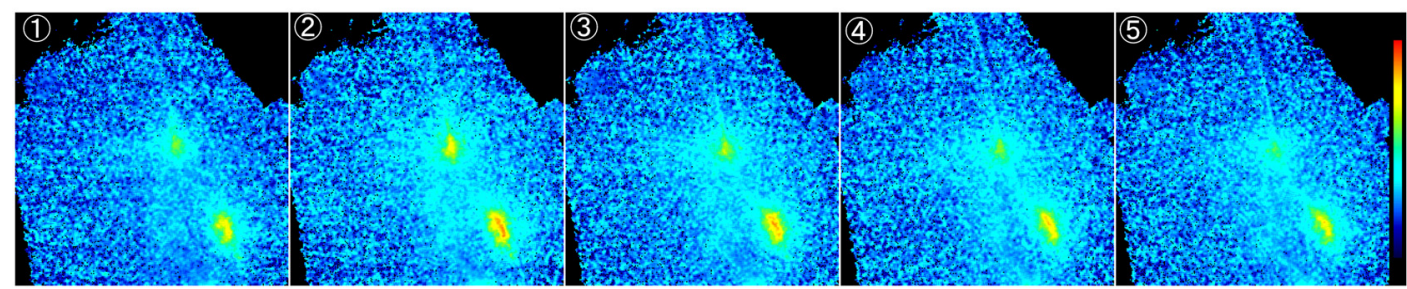

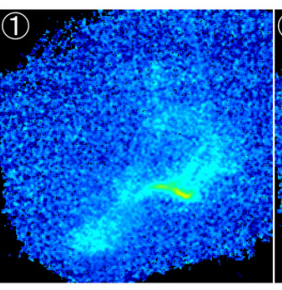

B
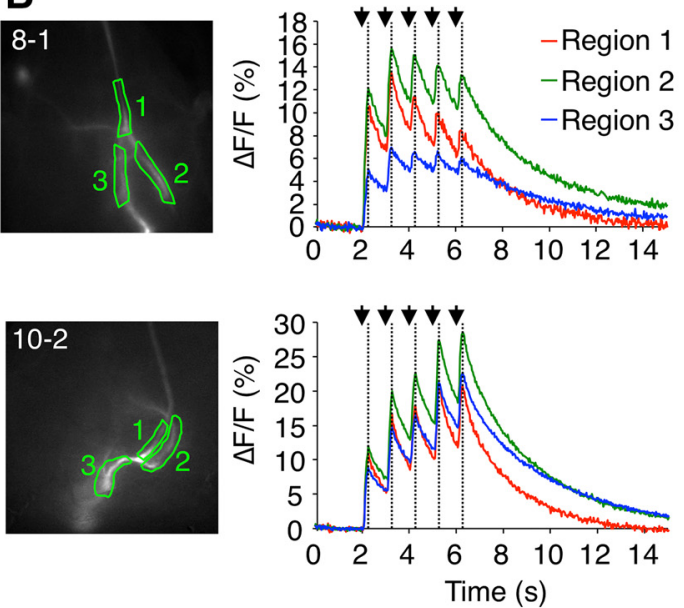

D

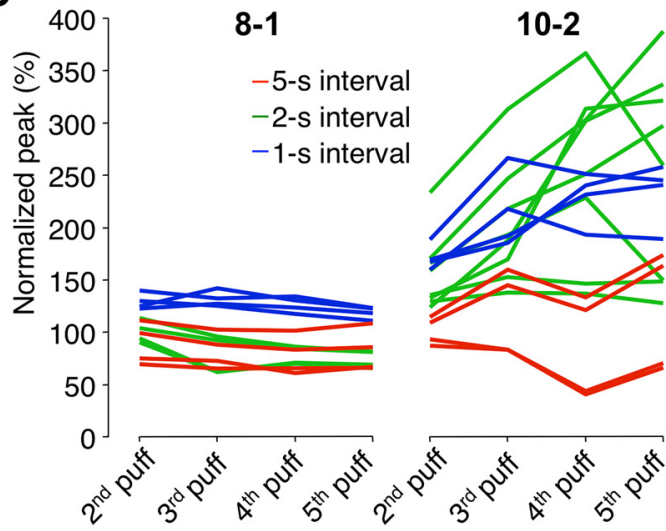

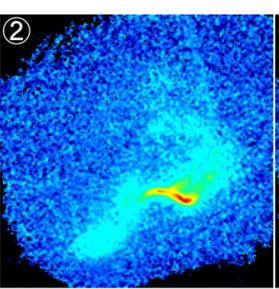

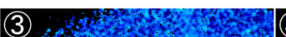

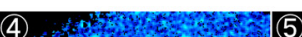

(5)

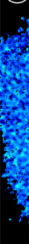

C
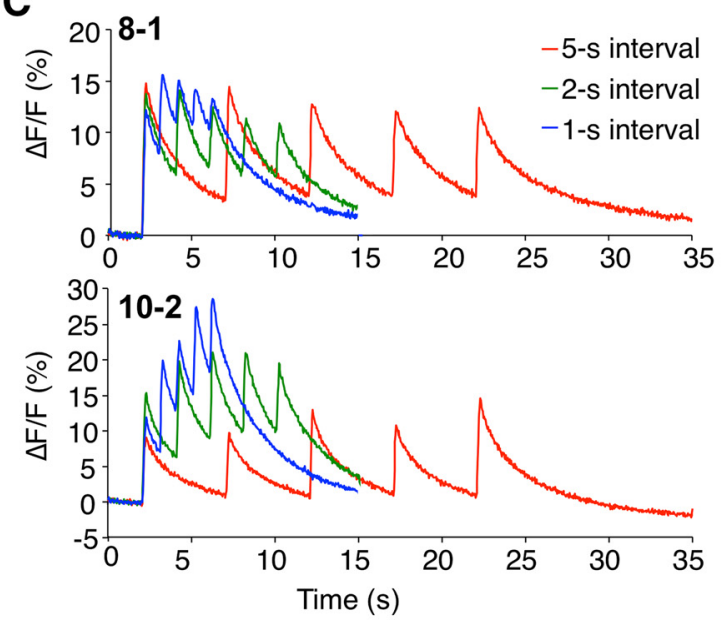

E

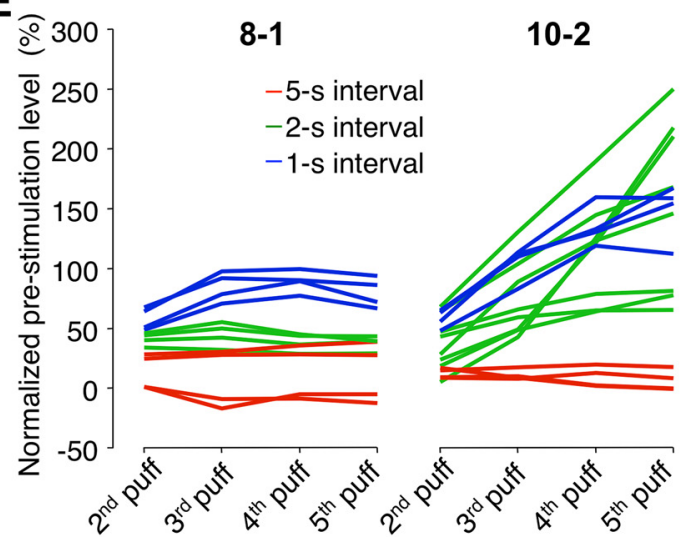

Figure 6. Dendritic $\mathrm{Ca}^{2+}$ dynamics in Gls during repetitive stimulation. $\boldsymbol{A}$, Pseudocolored images showing typical Ca ${ }^{2+}$ elevation in Gls 8-1 (top) and 10-2 (bottom). Images 1-5 were acquired at the peak of the $\mathrm{Ca}^{2+}$ response (indicated by dotted lines in $\boldsymbol{B}$ ) to each puff applied from the side contralateral to the soma at $1 \mathrm{~Hz}$. $\boldsymbol{B}$, Time course of $\mathrm{Ca}{ }^{2+}$ changes during a repetitive, $1 \mathrm{~Hz}$ stimulation. Three traces were obtained from three different ROIs (1-3), shown in the left images. Arrows indicate the timing of each stimulus. $\boldsymbol{C}$, Time course of the $\mathrm{Ca}^{2+}$ change in ROI 1 during repeated stimulation at various intervals. $\boldsymbol{D}, \boldsymbol{E}$, Changes in the peak value of transients $(\boldsymbol{D})$ and the prestimulation level $(\boldsymbol{E})$ of the $C \mathrm{a}^{2+}$ signal during four iterative puffs delivered at various intervals. Each value was scaled by the corresponding value for the response to the first puff. In $\mathrm{Gl} 10-2$, both the $\mathrm{Ca}^{2+}$ transient peak and the basal $\mathrm{Ca}{ }^{2+}$ level significantly increased depending on the stimulus order ( $p=1.18 \mathrm{E}-05$ and 9.45E-12 for peak and basal levels, respectively; 12 measurements from 4 ROls of 2 neurons; tw0-way repeated-measures ANOVA), but in GI 8-1, both aspects of the $\mathrm{Ca}^{2+}$ signal were reduced or unaltered ( $p=5.42 \mathrm{E}-06$ and 0.1121 for peak and basal levels, respectively; 16 measurements from 8 R0ls of 4 neurons).

To compare the directional tuning properties of $\mathrm{Ca}^{2+}$ responses across dendritic locations, we calculated mean angles based on the amplitude of the dendritic $\mathrm{Ca}^{2+}$ response for all ROIs and superimposed them on neuronal morphology as a heat map (Fig. 5D). In GI 8-1, the more distal regions of dendritic branches showed larger differences from one an- other in directional tuning and the differences gradually decreased from distal to proximal along the dendritic branch. In contrast, the dendritic $\mathrm{Ca}^{2+}$ responses in GI 10-2 showed similar directional preference throughout the dendrites. In other words, GI 8-1 displayed various spatial patterns of $\mathrm{Ca}^{2+}$ accumulation depending on the stimulus direction, whereas GI 
A

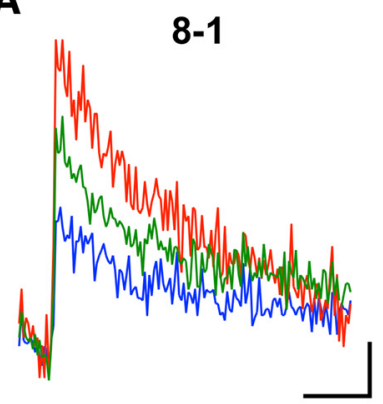

10-2

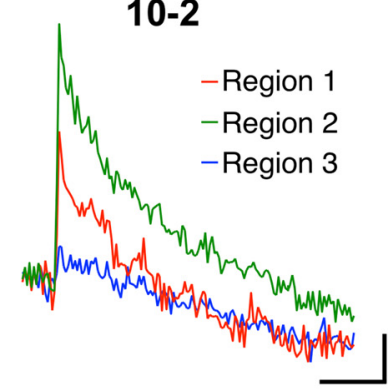

B

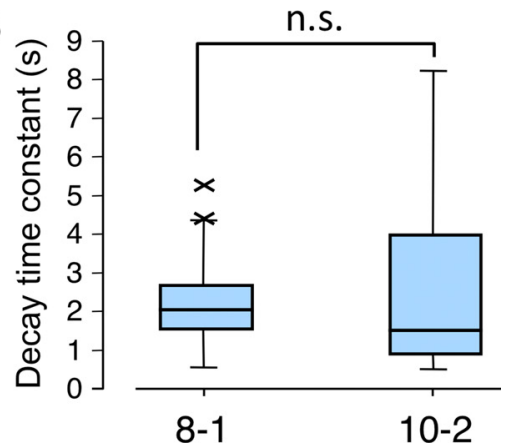

Figure 7. $\quad \mathrm{Ca}^{2+}$-buffering capacity in Gls. A, Time course of the typical $\mathrm{Ca}^{2+}$ transients induced by a single action potential in Gls 8-1 and 10-2. Fluorescence signals were measured in three regions corresponding to the initial segment and ipsilateral and contralateral dendrites for individual cells as indicated in the left images in Figure $6 B$. Vertical scale bars, $0.2 \%$ for $\mathrm{GI} 8-1$ and $0.5 \%$ for Gl 10-2 in $\Delta F / F$; horizontal scale bars, 1 s. B, Time constant of the $\mathrm{Ca}^{2+}$ decay in Gls 8-1 and 10-2. There was no difference in the $\mathrm{Ca}^{2+}$-buffering velocity between $\mathrm{Gl} 8$ - 1 and GI $10-2$ ( $p=0.3195$; 51 measurements from 24 ROls in 8 neurons for GI 8-1, 37 measurements from 20 ROls in 8 neurons for GI 10-2; Mann-Whitney $U$ test). The box plot properties are as described in the legend for Figure $4 D$.

10-2 displayed similar patterns of $\mathrm{Ca}^{2+}$ accumulation regardless of the stimulus direction.

\section{Different spatiotemporal patterns of $\mathrm{Ca}^{2+}$ accumulation between GIs}

We next focused on the spatial and temporal dynamics of $\mathrm{Ca}^{2+}$ accumulation over the dendrites of GIs $8-1$ and 10-2 during repeated stimulation (Fig. 6A,B). For GI 8-1, the peak values of transient increase in $\mathrm{Ca}^{2+}$ were nearly constant throughout stimulus delivery (Fig. 6B, top) and high $\mathrm{Ca}^{2+}$ levels were restricted to local regions of dendrites (Fig. 6A, top). In contrast, for GI 10-2, $\mathrm{Ca}^{2+}$ increases evoked by each puff accumulated in a stepwise fashion (Fig. 6B, bottom) and extended over the whole dendritic arbor (Fig. 6A, bottom). Differences in the temporal profiles of $\mathrm{Ca}^{2+}$ accumulation were more obvious in responses to puffs applied at various intervals (Fig. $6 C$ ). The peak value of each $\mathrm{Ca}^{2+}$ transient did not vary with the interpuff interval in GI 8-1, whereas shorter intervals between stimuli induced greater $\mathrm{Ca}^{2+}$ accumulation in GI 10-2. The dendritic $\mathrm{Ca}^{2+}$ response in GI 10-2 thus gradually increased both in terms of the $\mathrm{Ca}^{2+}$ transient peak and the basal $\mathrm{Ca}^{2+}$ level preceding the next puff with successive stimulus application (Fig. $6 D, E ; p=1.18 \mathrm{E}-05$ and $9.45 \mathrm{E}-12$ for peak and prestimulation levels, respectively, two-way repeatedmeasures ANOVA). In contrast, both of these aspects of the $\mathrm{Ca}^{2+}$ signal in GI 8-1 were less pronounced or unaltered ( $p=5.42 \mathrm{E}-06$ and 0.1121 for peak and prestimulation levels, respectively). In both types of GIs, the dendritic $\mathrm{Ca}^{2+}$ accumulation was higher in peak value and the prestimulation level at the shorter stimulus intervals $(p=0.0352,0.0004,0.0022$, and 3.15E-05 for the peak and prestimulation levels in GIs 8-1 and 10-2, respectively). The $\mathrm{Ca}^{2+}$-imaging results confirmed that repeated stimulation induced a large $\mathrm{Ca}^{2+}$ accumulation in GI 10-2 and maintained a high $\mathrm{Ca}^{2+}$ level throughout the dendrites. In contrast, each transient rise in $\mathrm{Ca}^{2+}$ declined rapidly in GI 8-1 and the area of $\mathrm{Ca}^{2+}$ accumulation was more localized in the dendritic arbor. There were, however, no significant differences between GIs 8-1 and 10-2 in decay time constants for the dendritic $\mathrm{Ca}^{2+}$ rise evoked by a single action potential (Fig. 7; $p=0.3195$, Mann-Whitney $U$ test), suggesting that both types of GI have a similar $\mathrm{Ca}^{2+}$ buffering capacity.

\section{Discussion}

In this study, repeated air puff stimulation delivered from a fixed angle induced direction-specific adaptation in wind-elicited walking behavior and firings in GIs, the key neurons for this behavior. Furthermore, iterative stimulation induced $\mathrm{Ca}^{2+}$ mediated short-term depression at sensory afferent-to-GI synapses. These results point to an SSA mechanism functioning at the intracellular, circuit, and behavioral levels. We reported previously that repeated puffs of air from a fixed angle temporarily reduce firing responses in GI 8-1 (Ogawa et al., 2001). Interestingly, some GIs, including 8-1, exhibited direction-specific adaptation in their responses, but others, such as 10-2, adapted to all stimulus directions regardless of the stimulus angle. The dendritic $\mathrm{Ca}^{2+}$ accumulation that leads to adaptation to repeated stimulation differed in its spatial distribution between GIs 8-1 and 10-2. Spatially localized $\mathrm{Ca}^{2+}$ signals in dendrites selectively regulate many postsynaptic reactions triggering modification of synaptic strength and structure (Augustine et al., 2003). Previous imaging studies have revealed that $\mathrm{Ca}^{2+}$ responses in distinct GI dendritic branches display unique directional tuning properties (Ogawa et al., 2004, 2008). In addition, GI 8-1 exhibited a variety of directional sensitivity in its $\mathrm{Ca}^{2+}$ responses depending on the dendrite location, suggesting that the dendritic $\mathrm{Ca}^{2+}$ signal is enhanced at synaptic sites activated by stimuli from a specific direction.

Based on both previous and present results, we propose the following neural mechanism underlying direction-specific adaptation in wind-elicited walking behavior in crickets. GI 8-1 (and possibly other types of GIs) generates fewer spikes in response to a short air puff than other GI types such as 10-2. Repeated stimulation therefore evokes transient increases in $\mathrm{Ca}^{2+}$ in dendrites, but these transients do not accumulate uniformly in GI 8-1 dendrites. Because the high $\mathrm{Ca}^{2+}$ concentration is spatially restricted, synaptic depression is induced only locally at specific synaptic sites, thereby reducing sensitivity to the direction of repetitive stimuli in GI 8-1. Oe and Ogawa (2013) reported that laser ablation of GI 8-1 reduced walking distance in the initial response to an air puff stimulus. GI 8-1 is likely involved in the maintenance of walking; therefore, the direction-specific attenuation induced by local $\mathrm{Ca}^{2+}$-mediated synaptic depression in GI 8-1 and other GIs would be expected to reduce walking in response to a stimulus from a specific direction.

In contrast, GI 10-2 responds with sustained, high-frequency spike bursts to air puff stimuli (Miller et al., 1991; Clague et al., 1997; Ogawa et al., 2008), which produce larger $\mathrm{Ca}^{2+}$ transients that accumulate and result in global $\mathrm{Ca}^{2+}$ elevation throughout the dendritic arbor. Furthermore, the dendritic $\mathrm{Ca}^{2+}$ signals in GI 10-2 are more homogeneous in their directional tuning, lead- 
ing to a similar distribution of the $\mathrm{Ca}^{2+}$ accumulation regardless of stimulus direction. Therefore, synaptic depression at all synaptic sites reduces sensitivity to all stimulus angles, resulting in little effect of repetitive stimulation on the directional tuning curves. This robustness and stability in the directional selectivity of GI 10-2 would ensure consistent encoding of air current information. A set of four interneurons (right and left GIs 10-2 and 10-3) accurately encodes the direction and velocity of airflow against a background of intrinsic adaptation and axonal conduction noise (Miller et al., 1991; Theunissen and Miller, 1991; Theunissen et al., 1996; Clague et al., 1997; Aldworth et al., 2012). Other GIs, such as 10-2, may not contribute to direct motor control of wind-elicited walking, but may instead convey sensory information for perceiving the location of a stimulus source to higher centers such as the brain and thoracic ganglia. Therefore, it is possible that selective synaptic depression induced by local $\mathrm{Ca}^{2+}$ accumulation in dendrites of key neurons directly involved in directional motor control underlies direction-specific behavioral adaptation, whereas some neurons that contribute to encoding the sensory information may be less affected by repetitive stimulation.

In other species of crickets and katydids, $\mathrm{Ca}^{2+}$ - and $\mathrm{Na}^{2+}{ }_{-}$ mediated $\mathrm{K}^{+}$channels are thought to contribute to acoustic processing and adaptation in auditory interneurons (Sobel and Tank, 1994; Baden and Hedwig, 2007; Triblehorn and Schul, 2013). It has also been reported that spike frequency adaptation in a visual neuron in the locust is mediated by a $\mathrm{Ca}^{2+}$-dependent $\mathrm{K}^{+}$conductance, which is similar to that associated with SK channels in vertebrates (Peron and Gabbiani, 2009). BAPTA injection into the dendrites of GIs suppressed the attenuation of the response to repeated puffs of air delivered at the 2 and $5 \mathrm{~s}$ intervals, but not at the $1 \mathrm{sinterval}$. This result provides evidence that the direction-specific adaptation could be mediated by dendritic $\mathrm{Ca}^{2+}$ accumulation. Adaptation to the frequent stimuli delivered at intervals of a few seconds was direction specific, but more frequent stimuli, such as at the $1 \mathrm{~s}$ interval, caused greater adaptation to all directions. It is likely that $\mathrm{Ca}^{2+}$-mediated modulation of $\mathrm{K}^{+}$channels regulates the immediate adaptation of spiking activity, which has a similar time course as the behavioral adaptation. Further, BAPTA affected the later phase of synaptic depression, during which the paired-pulse ratio did not change. Input resistance in dendrites of GIs is unaltered during shortterm depression induced by tetanic stimulation of cercal afferents (Ogawa et al., 2001), suggesting that the modulation of ligandgated channels by $\mathrm{Ca}^{2+}$ also mediate synaptic depression for adaptation in cricket GIs. The later phase $(60-80 \mathrm{~s}$ after the repetitive puffs) of $\mathrm{Ca}^{2+}$-dependent synaptic depression may depend on the other signaling cascades after an increase in $\mathrm{Ca}^{2+}$ concentration, which may regulate the modification of ligandgated channels.

Conversely, neither the response attenuation nor the earlier phase (up to $40 \mathrm{~s}$ after the repetitive puffs) of the synaptic depression induced by the repetitive stimulation at $1 \mathrm{~s}$ intervals were affected by the BAPTA injection. This implies that the adaptation to more frequent stimuli also relies on other mechanisms such as modulation of inhibitory inputs and depletion of the neurotransmitter within the sensory afferents. The immediate, acute direction-nonspecific adaptation induced by highly frequent stimuli (such as at $1 \mathrm{~s}$ interval) probably results from decrement of transmitter release, whereas modulation of the inhibitory circuit may occur during the earlier phase of the synaptic depression. GI 8-1 receives inhibitory inputs from cercal afferents ipsilateral to its soma (Baba et al., 2001), so it is possible that modulation of inhibitory inputs to GIs via local circuits within the TAG is also related to direction-specific adaptation. For example, a process analogous to SSA was found in a cultured cortical neuron network using an oddball-like paradigm of electrical stimulation (Eytan et al., 2003). In this experiment, bicuculline, a blocker of GABA transmission, abolished the selective enhancement in response to a novel stimulus and it was proposed that adaptation of the inhibitory circuits led to SSA. In addition, adaptation to short visual stimulation reverses the directional preference of direction-selective retinal ganglion cells (Rivlin-Etzion et al., 2012). Therefore, even within a hardwired peripheral circuit, sensory processing for detecting features such as stimulus direction can be altered by repeated exposure to the same stimulus.

The present findings on direction-specific adaptation in the cricket mechanosensory system are the first illustration of subcellular adaptation mechanisms of specific neurons underlying behavioral SSA. A neural correlation between behavioral habituation and SSA has been observed in an owl's gaze control in response to auditory stimuli (Netser et al., 2011), but it is difficult to use SSA to describe individual neuronal responses to behavioral habituation in the more complex nervous systems of birds and mammals. In mammals, this phenomenon has been explained by differential adaptation to inputs by cells exhibiting SSA (May and Tiitinen, 2010). It was also proposed that SSA in the auditory cortex emerges from variable adaptation of channels that span the receptive field of neurons (Taaseh et al., 2011). $\mathrm{Ca}^{2+}$-mediated depression at local dendritic sites allows GIs to selectively reduce the response to input channels from a specific stimulus direction. In an imaging study on insect auditory interneuron, Prešern et al. (2015) reported that $\mathrm{Ca}^{2+}$ and $\mathrm{Na}^{+}$ accumulations resulting in frequency-specific adaptation were restricted within the stimulated region of the dendrite. Dendritic compartmentalization of such cationic signals may be a common basis for subcellular mechanisms of SSA in insect neurons.

Finally, differences in SSA between GIs 8-1 and 10-2 imply that the adaptation of these cells is directly correlated with the division of their roles in behavioral control. The direction-specific adaptation in GI 8-1 related to motor control in wind-elicited walking suggests that $\mathrm{Ca}^{2+}$-mediated depression underlies the habituation of specific behaviors. Although the ethological significance of these findings remains unclear, direction-specific adaptation may enable the cricket to detect approaching predators and accurately direct oriented escape behaviors under windy conditions. For all animals, including insects, detecting deviations from familiar or typical stimuli is a task of the sensory system that is essential for survival. SSA, which is basic to this task, likely occurs at various levels in sensory systems. Previous attempts to explain SSA in the mammalian brain described the modulation of circuits by the environment; the present study has demonstrated that SSA in an innate insect behavior relies on stimulus-dependent intracellular $\mathrm{Ca}^{2+}$ dynamics in key neurons, which prompts a reconsideration of SSA in complex systems.

\section{References}

Aldworth ZN, Bender JA, Miller JP (2012) Information transmission in cercal giant interneurons is unaffected by axonal conduction noise. PLoS One 7:e30115. CrossRef Medline

Augustine GJ, Santamaria F, Tanaka K (2003) Local calcium signaling in neurons. Neuron 40:331-346. CrossRef Medline

Baba Y, Masuda H, Shimozawa T (2001) Proportional inhibition in the cricket medial giant interneuron. J Comp Physiol A 187:19-25. CrossRef Medline

Baden T, Hedwig B (2007) Neurite specific $\mathrm{Ca}^{2+}$ dynamics underlying sound processing in an auditory interneurone. Dev Neurobiol 67:68-80. Medline 
Clague H, Theunissen F, Miller JP (1997) Effects of adaptation on neural coding by primary sensory interneurons in the cricket cercal system. J Neurophysiol 77:207-220. Medline

Condon CD, Weinberger NM (1991) Habituation produces frequency specific plasticity of receptive fields in the auditory cortex. Behav Neurosci 105:416-430. CrossRef Medline

Derdikman D, Yu C, Haidarliu S, Bagdasarian K, Arieli A, Ahissar E (2006) Layer specific touch-dependent facilitation and depression in the somatosensory cortex during active whisking. J Neurosci 26:9538-9547. CrossRef Medline

Dragoi V, Sharma J, Sur M (2000) Adaptation-induced plasticity of orientation tuning in adult visual cortex. Neuron 28:287-298. CrossRef Medline

Dragoi V, Rivadulla C, Sur M (2001) Foci of orientation plasticity in visual cortex. Nature 411:80-86. CrossRef Medline

Eytan D, Brenner N, Marom S (2003) Selective adaptation in networks of cortical neurons. J Neurosci 23:9349-9356. Medline

Givois V, Pollack GS (2000) Sensory habituation of auditory receptor neurons: implications for sound localization. J Exp Biol 203:2529-2537. Medline

Gras H, Kohstall D (1998) Current injection into interneurones of the terminal ganglion modifies turning behaviour of walking crickets. J Comp Physiol A 182:351-361. CrossRef

Hirota K, Sonoda Y, Baba Y, Yamaguchi T (1993) Distinction in morphology and behavioral role between dorsal and ventral groups of cricket giant interneurons. Zool Sci 10:705-709.

Jacobs GA, Murphey RK (1987) Segmental origins of the cricket giant interneuron system. J Comp Neurol 265:145-157. CrossRef Medline

Jacobs GA, Miller JP, Aldworth Z (2008) Computational mechanisms of mechanosensory processing in the cricket. J Exp Biol 211:1819-1828. CrossRef Medline

Katz Y, Heiss JE, Lampl I (2006) Cross-whisker adaptation of neurons in the rat barrel cortex. J Neurosci 26:13363-13372. CrossRef Medline

Landolfa M, Miller JP (1995) Stimulus-response properties of cricket cercal filiform receptors. J Comp Physiol A 177:749-757.

Malone BJ, Scott BH, Semple MN (2002) Context-dependent adaptive coding of interaural phase disparity in the auditory cortex of awake macaques. J Neurosci 22:4625-4638. Medline

May PJ, Tiitinen H (2010) Mismatch negativity (MMN), the devianceelicited auditory deflection, explained. Psychophysiology 47:66-122. CrossRef Medline

Miller JP, Jacobs GA, Theunissen FE (1991) Representation of sensory information in the cricket cercal sensory system. I. Response properties of the primary interneurons. J Neurophysiol 66:1680-1689. Medline

Miller JP, Krueger S, Heys JJ, Gedeon T (2011) Quantitative characterization of the filiform mechanosensory hair array on the cricket cercus. PLoS One 6:e27873. CrossRef Medline

Movshon JA, Lennie P (1979) Pattern selective adaptation in visual cortical neurones. Nature 278:850-852. CrossRef Medline

Nelken I, Ulanovsky N (2007) Mismatch negativity and stimulus specific adaptation in animal models. J Psychophysiol 21:214-223. CrossRef

Netser S, Zahar Y, Gutfreund Y (2011) Stimulus specific adaptation: Can it be a neural correlate of behavioral habituation? J Neurosci 31:1781117820. CrossRef Medline

Oe M, Ogawa H (2013) Neural basis of stimulus-angle-dependent motor control of wind-elicited walking behavior in the cricket Gryllus bimaculatus. PLoS One 8:e80184. CrossRef Medline

Ogawa H, Baba Y, Oka K (2001) Dendritic calcium accumulation regulates wind sensitivity via short-term depression at cercal sensory-to giant interneuron synapses in the cricket. J Neurobiol 46:301-313. Medline

Ogawa H, Baba Y, Oka K (2004) Directional sensitivity of dendritic calcium responses to wind stimuli in the cricket giant interneuron. Neurosci Lett 358:185-188. CrossRef Medline
Ogawa H, Cummins GI, Jacobs GA, Oka K (2008) Dendritic design implements algorithm for synaptic extraction of sensory information. J Neurosci 28:4592-4603. CrossRef Medline

Palka J, Levine R, Schubiger M (1977) The cercus-to-giant interneuron system of crickets. I. Some attributes of the sensory cells. J Comp Physiol A 119:267-283. CrossRef

Peron S, Gabbiani F (2009) Spike frequency adaptation mediates looming stimulus selectivity in a collision-detecting neuron. Nat Neurosci 12:318326. CrossRef Medline

Ponnath A, Hoke KL, Farris HE (2013) Stimulus change detection in phasic auditory units in the frog midbrain: frequency and ear specific adaptation. J Comp Physiol A 199:295-313. CrossRef Medline

Prešern J, Triblehorn JD, Schul J (2015) Dynamic dendritic compartmentalization underlies stimulus-specific adaptation in an insect neuron. J Neurophysiol 113:3787-3797. CrossRef Medline

Rankin CH, Abrams T, Barry RJ, Bhatnagar S, Clayton DF, Colombo J, Coppola G, Geyer MA, Glanzman DL, Marsland S, McSweeney FK, Wilson DA, Wu CF, Thompson RF (2009) Habituation revisited: an updated and revised description of the behavioral characteristics of habituation. Neurobiol Learn Mem 92:135-138. CrossRef Medline

Reches A, Gutfreund Y (2008) Stimulus specific adaptations in the gaze control system of the barn owl. J Neurosci 28:1523-1533. CrossRef Medline

Ringo JL (1996) Stimulus specific adaptation in inferior temporal and medial temporal cortex of the monkey. Behav Brain Res 76:191-197. Medline

Rivlin-Etzion M, Wei W, Feller MB (2012) Visual stimulation reverses the directional preference of direction selective retinal ganglion cells. Neuron 76:518-525. CrossRef Medline

Saul AB, Cynader MS (1989) Adaptation in single units in visual cortex: the tuning of aftereffects in the spatial domain. Vis Neurosci 2:593-607. CrossRef Medline

Schul J, Sheridan RA (2006) Auditory stream segregation in an insect. Neuroscience 138:1-4. CrossRef Medline

Schul J, Mayo AM, Triblehorn JD (2012) Auditory change detection by a single neuron in an insect. J Comp Physiol A 198:695-704. CrossRef Medline

Shimozawa T, Kanou M (1984) The aerodynamics and sensory physiology of range fractionation in the cercal filiform sensilla of the cricket Gryllus bimaculatus. J Comp Physiol A 155:495-505. CrossRef

Sobel EC, Tank DW (1994) In vivo $\mathrm{Ca}^{2+}$ dynamics in a cricket auditory neuron: an example of chemical computation. Science 263:823-826. CrossRef Medline

Taaseh N, Yaron A, Nelken I (2011) Stimulus specific adaptation and deviance detection in the rat auditory cortex. PLoS One 6:e23369. CrossRef Medline

Theunissen FE, Miller JP (1991) Representation of sensory information in the cricket cercal sensory system. II. Information theoretic calculation of system accuracy and optimal tuning-curve widths of four primary interneurons. J Neurophysiol 66:1690-1703. Medline

Theunissen F, Roddey JC, Stufflebeam S, Clague H, Miller JP (1996) Information theoretic analysis of dynamical encoding by four identified primary sensory interneurons in the cricket cercal system. J Neurophysiol 75:1345-1364. Medline

Triblehorn JD, Schul J (2013) Dendritic mechanisms contribute to stimulus specific adaptation in an insect neuron. J Neurophysiol 110:2217-2226. CrossRef Medline

Ulanovsky N, Las L, Nelken I (2003) Processing of low-probability sounds by cortical neurons. Nat Neurosci 6:391-398. CrossRef Medline

Zar JH (2010) Biostatistical analysis, Ed 5, pp 605-668. Upper Saddle River, NJ: Prentice-Hall.

Zucker RS (1999) Calcium- and activity-dependent synaptic plasticity. Curr Opin Neurobiol 9:305-313. CrossRef Medline 